\title{
Superior Mesenteric Artery Syndrome: A High Index of Suspicion
}

\author{
Ahmed Arifur Rahman
}

\begin{abstract}
Duodenal obstruction by compression from the superior mesenteric artery (SMA) can be managed using minimally invasive techniques. We will discuss a presentation of a 46-year-old female with abdominal pain and vomiting on background of recent weight loss. Diagnosis was made with CT of abdomen and barium follow-through. She was treated with surgical intervention as initial conservative management failed. Surgery is required when conservative management fails. Though Strong procedure is easy and effective for some patients, duodenojejunostomy is the treatment of choice. Advances in minimally invasive surgery have demonstrated the safety and low morbidity of laparoscopically created enteric anastomoses.
\end{abstract}

Keywords: SMA syndrome; Strong procedure; Wilkie's syndrome

\section{Introduction}

Superior mesenteric artery (SMA) syndrome is an interesting phenomenon that occurs when the SMA compresses the third part of the duodenum. This is rare and much of the literature pertains to case reports and small case series. Its wide range of predisposing factors and mild chronic obstructive features make the diagnosis a high index of suspicion. It was first described in 1842 by Rokitansky and the first case series of 75 patients was published by Wilkie in 1927.

\section{Case Report}

A 46-year-old female presented to emergency department with 2 days history of abdominal pain and vomiting. Initially she developed sharp, stabbing generalized abdominal pain followed by abdominal distension. She did not open her bowel for 4 days with nil flatus for 2 days. She had previously been treated for gastritis for $H$. pylori 15 years ago. She never had any abdomi-

Manuscript submitted December 8, 2017, accepted December 20, 2017

St George Hospital, Gray Street, Kogarah, NSW, Australia.

Email: arif_ssmc@yahoo.com

doi: https://doi.org/10.14740/jmc2982w nal operation. She also noticed a $50 \mathrm{~kg}$ weight loss in last 1 year, some of which was intentional. Since dieting, her bowel motion reduced from three times per day to once per week. On physical examination, she was hemodynamically stable. Her abdomen was soft but generalized tender with no bowel sound. CT scan of abdomen showed dilatation of stomach, first and second part of duodenum. There was compression of D3 (third part of duodenum) between the aorta and SMA (Figs. 1 and 2). Distal to this, the bowel loops were completely collapsed. A subsequent barium meal and follow-through demonstrated a linear compression at the third part of the duodenum causing dilation of the proximal duodenum. This was also compatible with compression by the SMA (Fig. 3a-d). Upper gastrointestinal endoscopy was organized that showed gastroparesis and scattered stomach ulcer in antrum. D1 and D2 were visualized without any obvious lesion. It was unable to reach D3, possibly secondary to dilated and stretched stomach. SMA syndrome was diagnosed. She was treated conservatively for 6 days without any improvement. She was taken to theater and Strong procedure was performed and discharged on second postoperative

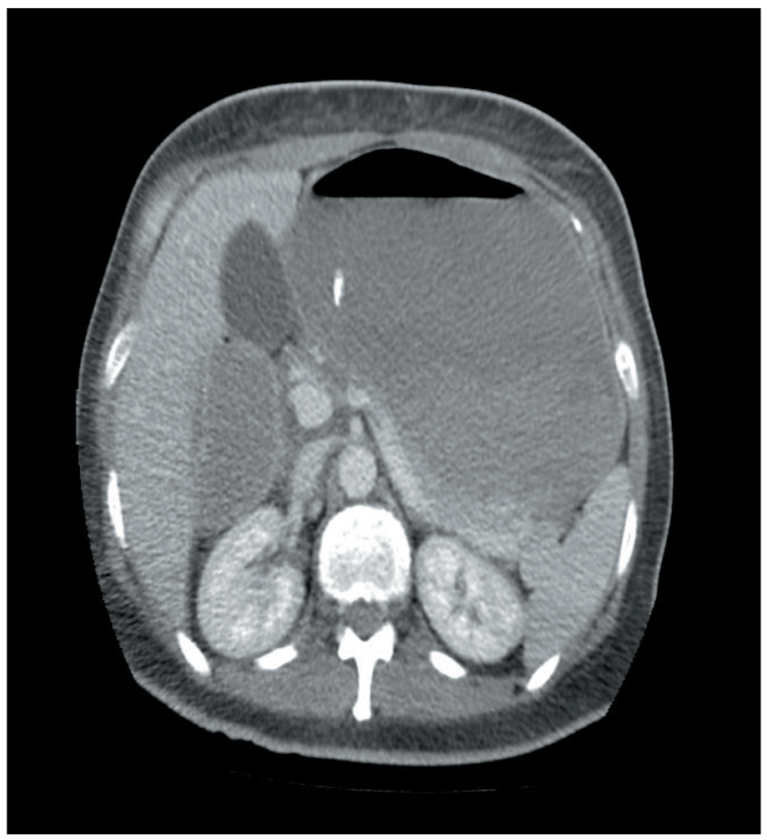

Figure 1. CT scan of abdomen showing dilatation of stomach, first and second part of duodenum. There was compression of D3 (third part of duodenum) between the aorta and SMA. 


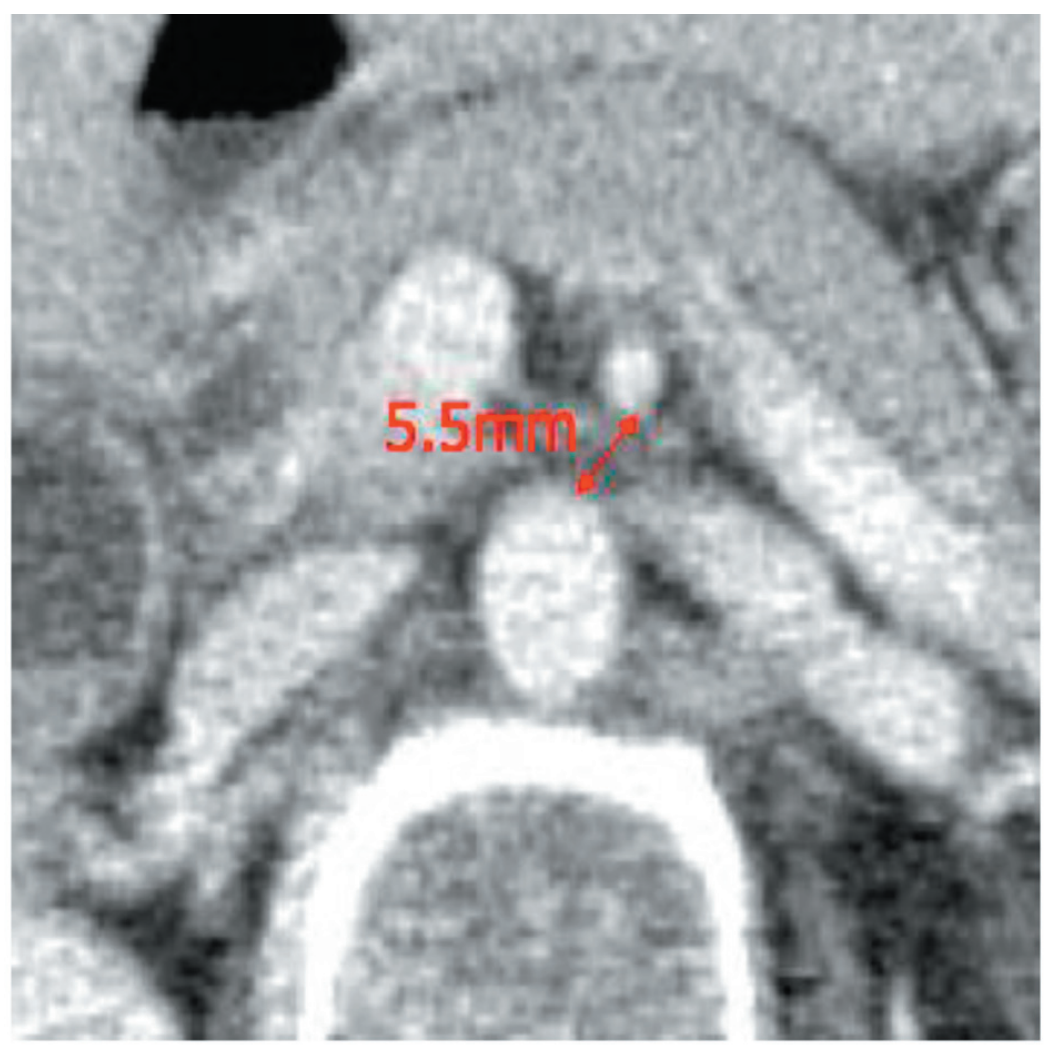

Figure 2. CT scan of abdomen showing decreased aorto-mesenteric distance.

day. Postoperatively, she was put on a high-calorie diet, and subsequently gained $10 \mathrm{~kg}$ in body weight with no recurrence on 6 and 12 months follow-up.

\section{Discussion}

SMA syndrome, also known as aortomesenteric duodenal compression or duodenal vascular compression or Wilkie's syndrome or Cast syndrome, is a rare clinical entity that results from the compression of the third part of the duodenum as it passes between the overlying SMA and the aorta [1]. Prevalence is about $0.013-0.3 \%$ with female predominance and occurs mostly between 10 and 39 years.

Normally, the SMA forms an approximately $45^{\circ}$ angle (range, $38-65^{\circ}$ ) as it comes off the aorta. This angulation produces an aortomesenteric distance that spans $10-28 \mathrm{~mm}$ without impingement on the duodenal lumen as the third portion traverses laterally toward the ligament of Treitz. Decreasing the aortomesenteric angle to less than $25^{\circ}$ will decrease the aortomesenteric distance to less than $10 \mathrm{~mm}$. When this condition occurs, the third portion of the duodenum is compressed and SMA syndrome symptoms ensue $[2,3]$.

\section{Predisposing factors}

Predisposing factors include: 1) reapid weight loss; 2) reduc- tion of the mesenteric fat around the SMA (causes: AIDS, malabsorption, cancer, cerebral palsy, and other conditions associated with cachexia; catabolic conditions, e.g. burns; eating disorders, e.g. anorexia nervosa and drug abuse [1]); 3) following surgery (spine surgery: scoliosis correction, due to a relative lengthening of the spine postoperatively (prevalence 0.5-2.4\%) [4]; ileal pouch-anal anastomosis (IPAA) - stretch the SMA over duodenum as the ileal pouch reaches pelvis; surgery associated with rapid weight loss - bariatric surgery, esophagectomy, abdominal trauma; others: compression from an AAA or SMA aneurysm [4]); and 4) anatomical variants (high ligament of Treitz; low origin of the SMA).

\section{Diagnosis}

Diagnosis requires a high index of suspicion. Unlikely our patient, most cases present in a chronic, insidious manner with the development of intermittent abdominal pain associated with vomiting, early satiety, and anorexia, esophageal reflux or anorexia. Sometimes they present with weight loss. Patients rarely present with signs and symptoms of acute duodenal obstruction [5].

\section{Investigations}

Radiological evidence of D3 compression by SMA includes: 1) aorto-mesenteric angle $<22^{\circ}$ (normal $38-65^{\circ}$ ) [6]; 2) aor- 


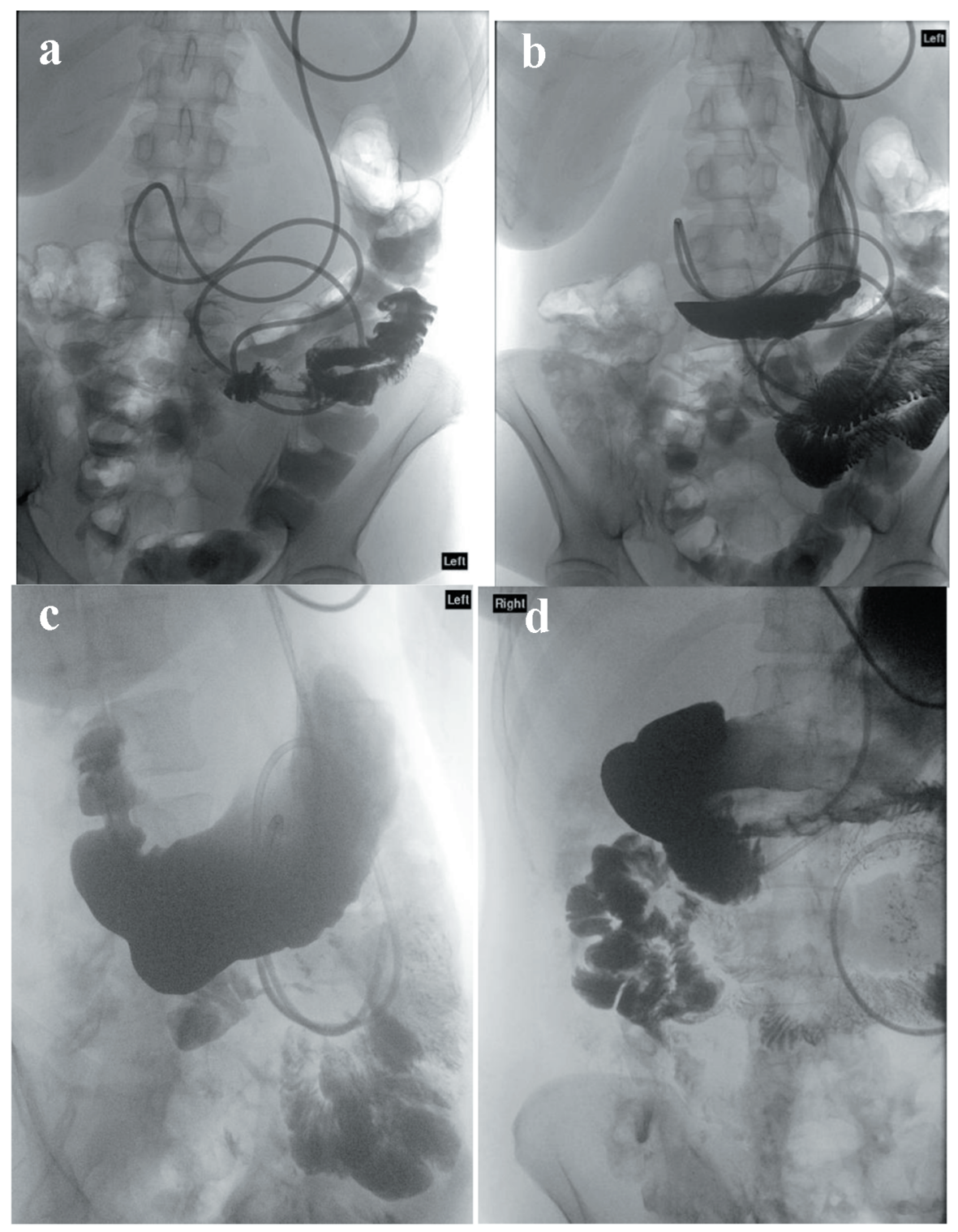

Figure 3. Barium meal follow-through demonstrating a linear compression at the third part of the duodenum causing dilation of the proximal duodenum. This was also compatible with compression by the SMA.

to-mesenteric distance $<8 \mathrm{~mm}$ (normal $10-28 \mathrm{~mm}$ ); and 3) proximal duodenal dilation with cut-off at D3 indicates SMA syndrome (Fig. 4a, b).

Contrast X-ray studies involve barium studies (that show duodenal dilation, retention of barium within the duodenum, and the characteristic vertical linear extrinsic compression at the third part of the duodenum), CT of abdomen (with oral contrast) and CT angiogram [7]. Endoscopy is used to confirm external compression at the third part of the duodenum and can rule out intrinsic pathology of the duodenum [6].

\section{Treatment}

Conservative management combined with a high-calorie diet is usually advocated $[1,6]$. Nutritional support with nasojejunal feeds when possible or TPN in selected patients is helpful. Positioning the patient in a knee-to-chest position or prone after eating helps to improve symptoms.

Surgical intervention is indicated only when medical therapy fails. Strong procedure involves division of the ligament of Treitz [3], and allows caudal duodenal mobilization outside the aortomesenteric angle. Although appealing because bowel integrity is not compromised by an anastomosis, the drawback of the Strong procedure is a $25 \%$ failure rate resulting from tethering of the inferior pancreaticoduodenal artery [2]. Bypass of the third part of the duodenum by the creation of a duodenojejunostomy together with division of the ligament of Treitz has been the operation of choice. Recently, laparoscopic duodenojejunostomy 

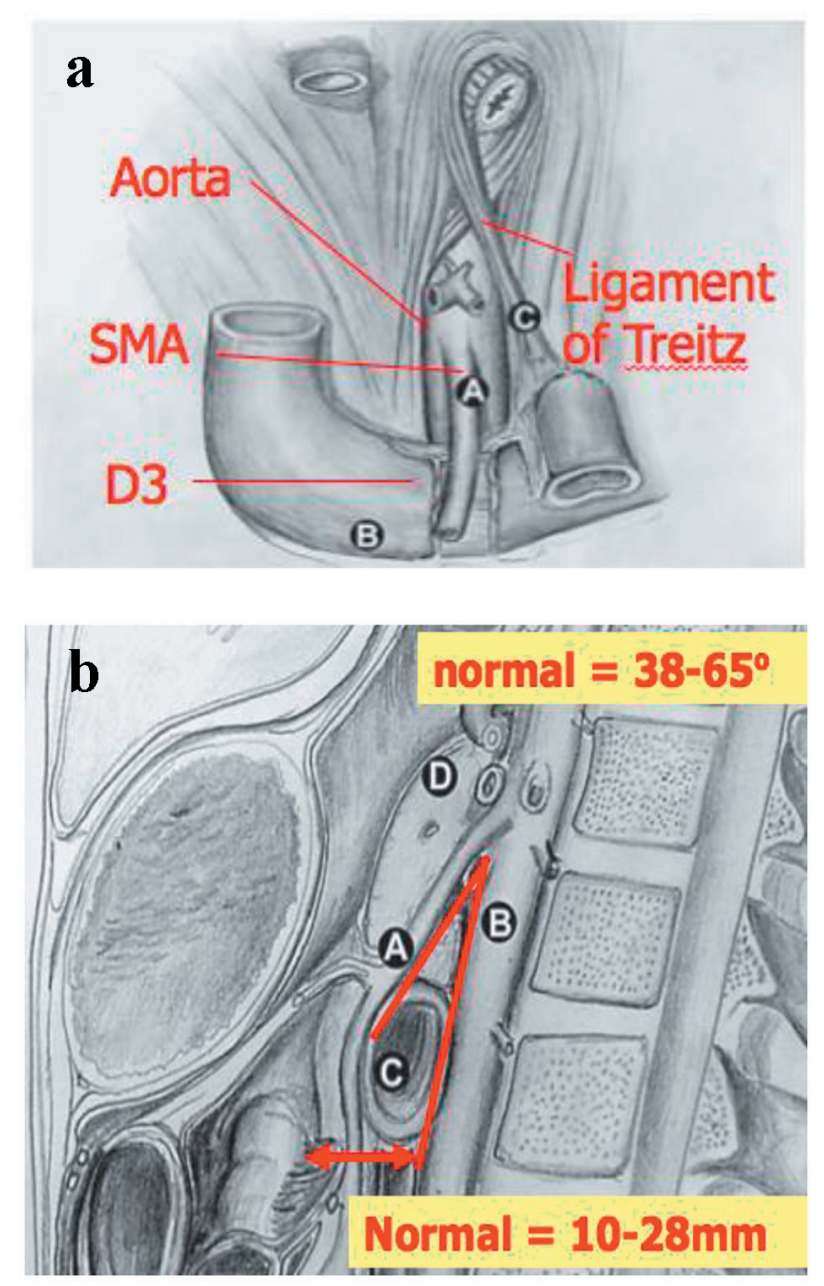

Figure 4. (a) Anatomy and origin of SMA. (b) Normal aorto-mesenteric angle and distance.

has been attempted in a number of centers with satisfactory results [1].

\section{Conclusions}

SMA syndrome is a rare cause of chronic duodenal obstruction. Diagnosis requires a high index of suspicion. It requires surgical intervention if conservative management fails. Currently, advances in minimal-access bariatric surgery have demonstrated the safety and low morbidity of laparoscopically created enteric anastomoses [3]. But there is no large scale study to compare the treatment modalities.

\section{Conflict of Interest}

There is no conflict of interest.

\section{References}

1. Kwan E, Lau H, Lee F. Wilkie's syndrome. Surgery. 2004;135(2):225-227.

2. Mandarry MT, Zhao L, Zhang C, Wei ZQ. A comprehensive review of superior mesenteric artery syndrome. Acta Chirurgica Austriaca. 2010;42(5):229-236.

3. Pottorf BJ, Husain FA, Hollis HW, Jr., Lin E. Laparoscopic management of duodenal obstruction resulting from superior mesenteric artery syndrome. JAMA Surg. 2014;149(12):1319-1322.

4. Ahmed AR, Taylor I. Superior mesenteric artery syndrome. Postgrad Med J. 1997;73(866):776-778.

5. Baltazar U, Dunn J, Floresguerra C, Schmidt L, Browder W. Superior mesenteric artery syndrome: an uncommon cause of intestinal obstruction. South Med J. 2000;93(6):606-608.

6. Ylinen P, Kinnunen J, Hockerstedt K. Superior mesenteric artery syndrome. A follow-up study of 16 operated patients. J Clin Gastroenterol. 1989;11(4):386-391.

7. Ooi GC, Chan KL, Ko KF, Peh WC. Computed tomography of the superior mesenteric artery syndrome. Clin Imaging. 1997;21(3):210-212. 